\title{
Colin a le front de venir à cette réunion Une construction avec nom de partie du corps
}

\author{
Thomas Bertin \\ Dylis (Université de Rouen) 7, rue Thomas Becket 76830 Mont-Saint-Aignan Cedex (France)
}

\begin{abstract}
Résumé. Cette étude prolonge un travail de recherche sur la signification des noms des parties du corps humain $\left(\mathrm{N}_{\mathrm{pc}}\right)$ (Bertin, 2018). On s'intéresse à la construction illustrée par Colin a le front de venir à cette réunion (NPV). Après un inventaire des $\mathrm{N}_{\mathrm{pc}}$ compatibles avec NPV, on procède à une analyse sémantique : d'une part, on cerne le sens de NPV (en se focalisant sur la contribution du $\mathrm{N}_{\mathrm{pc}}$ ) et, d'autre part, on tire profit de cette première étape pour l'analyse sémantique des $\mathrm{N}_{\mathrm{pc}}$ eux-mêmes.
\end{abstract}

\begin{abstract}
Colin a le front de venir à cette réunion. A body-part term $\left(\mathbf{N}_{\mathbf{p c}}\right)$ construction. This study follows a research on $\mathrm{N}_{\mathrm{pc}}$ semantics (Bertin, 2018). We deal with utterances such as Colin a le front de venir à cette réunion. At first, we identify $\mathrm{N}_{\mathrm{pc}}$ fitting this construction. Then, we figure out its meaning with a focus on the $\mathrm{N}_{\mathrm{pc}}$ semantic contribution. At last, we give an insight into consequences on body-part terms semantic analysis.
\end{abstract}

Cette étude ${ }^{1}$ s'inscrit dans un projet plus vaste de description des « noms de parties du corps humain » en français (souvent très polysémiques ${ }^{2}$ ). Pour un nom, il s'agit de circonscrire une identité sémantique. Nous envisageons ainsi le projet de la sémantique dont Larrivée résume le "cahier des charges » en ces termes : "La discipline doit pouvoir dire ce que signifie un signe au regard de la tension entre l'unité qui le caractérise par opposition aux autres signes, et la diversité de ses manifestations contextuelles » (2008: 4). Face au problème de la «polysémie contextuelle généralisée » (Gosselin, 1996), une piste possible - susceptible de résoudre l'apparente contradiction entre principes de compositionnalité et de contextualité - est de distinguer « une signification abstraite et invariable, associée au marqueur [...], et ses significations en contexte » (1996:100). Cette signification pourrait rendre compte d'une unité par-delà la diversité (Larrivée), donc d'une identité sémantique.

Dans cette perspective nous avons proposé une analyse des noms artère, bouche, cœur, épaule et pied (Bertin, 2018) sans ignorer que, dans le domaine nominal, en particulier avec des noms dont le sens premier est réputé concret, la mise au jour d'une signification invariante subsumant une diversité d'emplois est sujette à caution (cf. Kleiber, 1999).

Nous nous focaliserons ici sur la construction exemplifiée par Colin a le front de venir à cette réunion dans le but d'alimenter la réflexion sur l'identité sémantique de certains noms. Pour cela, nous délimiterons d'abord notre objet empirique (section 1). Ensuite, nous ferons un état des lieux des instanciations possibles pour cette construction (section 2). Enfin, on développera l'analyse sémantique proprement dite (section 3). 


\section{Délimitation de l'objet empirique}

\subsection{Des « noms de parties du corps humain »?}

A l'instar des noms de propriétés ou d'états psychologiques (Anscombre, 1995), la classe des noms de parties du corps humain, si elle semble intuitivement fondée, n'est pas si facile à définir linguistiquement. Toutefois, « on admet généralement l'existence d'une classe sémantique, bien délimitée syntaxiquement, correspondant intuitivement à la catégorie des "parties du corps humain" » (Leeman, 2005: 189) en s'appuyant sur les structures de possession inaliénable (Chapell \& McGregor, 1996 ; Van Petegehm, 2006).

Dans Bertin (2018 : 93), nous avons complété ces critères syntaxiques, notamment par des critères de dénomination terminologique, pour définir un "nom de partie du corps humain » en français (désormais $\mathrm{N}_{\mathrm{pc}}$ ). Puis, sur des critères essentiellement lexicographiques, nous avons constitué un corpus de $77 \mathrm{~N}_{\text {pc }}$ polysémiques (2018:449).

C'est ce corpus que nous utilisons pour tester la construction décrite ci-dessous.

\subsection{La construction NPV}

Comme indiqué précédemment, nous partons de la construction exemplifiée par :

1. Colin a le front de venir à cette réunion

D'un point de vue morphosyntaxique, on peut considérer cette construction comme une périphrase verbale constituée de deux éléments verbaux (Gosselin, sous presse). Le premier élément, une locution verbale (avoir le front de), fonctionne comme un opérateur. Il est en effet impossible de substituer une complétive à l'infinitif (Gross, 1999: 8 ; Lamiroy, 1999: 38): *Colin a le front que je/il vienne à cette réunion (vs. Colin a la volonté de venir / que je vienne à cette réunion). Le second élément, à l'infinitif (venir), est en emploi prédicatif.

D'un point de vue lexical, ce qui attire l'attention, c'est le recours à un $\mathrm{N}_{\mathrm{pc}}$ (ici, front) ${ }^{3}$ dont l'emploi ne correspond précisément pas au sens «partie du corps ». Nous étudions ainsi la construction avoir $L E N_{p c} P R E P V_{\text {inf }}$ où $L E$ désigne l'article défini, PREP une préposition (pas forcément de, on le verra) et $V_{\text {inf }}$ un verbe à l'infinitif. Dans la suite, cette construction est simplement notée NPV.

Précisons que, dans la perspective théorique que nous avons explicitée en introduction, une attention portée à la construction NPV est doublement motivée. D'une part, à l'image de la construction exemplifiée par rire à gorge déployée ou traverser à pied sec, NPV est "productive » et il est tentant de chercher à comprendre le rôle sémantique que joue le $\mathrm{N}_{\mathrm{pc}}$ dans cette configuration syntaxique (3.2). D'autre part, il semble pertinent d'envisager les caractéristiques sémantiques qui émergent de la compatibilité d'un $\mathrm{N}_{\mathrm{pc}}$ avec NPV (3.3).

\section{Noms compatibles avec NPV : état des lieux}

Cette deuxième section, à vocation descriptive, propose un inventaire des instanciations possibles de NPV. Il s'agit de repérer les $\mathrm{N}_{\mathrm{pc}}$ compatibles avec NPV tout en repérant la nature de la préposition, le nombre du déterminant et une éventuelle polarité négative.

Nous exploitons deux bases de données différentes - accessibles en ligne, munies d'outils de recherche avancée : le corpus contemporain de Frantext (Fx) et la base frWaC (fW). La base Fx regroupe des textes écrits après 1980 et rassemble plus de 36 millions de mots. Dans le cadre de cette étude, elle présente le "défaut» de compiler des textes majoritairement littéraires parfois éloignés de la langue courante (écrite ou orale). 
C'est pourquoi fW est une base complémentaire intéressante. Portée par la plateforme NoSketch Engine, celle-ci regroupe un vaste ensemble de pages Web (en français) et contient plus de 1,3 milliards de mots. Une base de données de ce type offre «a unique viewpoint into uncensored current usage » (Bouveret et Sweetser, $2009: 3$ ).

Chacun des $77 \mathrm{~N}_{\mathrm{pc}}$ du corpus de référence a été testé dans Fx et fW avec NPV. Cidessous, seuls les noms dont au moins une occurrence a été trouvée sont signalés (les autres sont donc considérés comme a priori $^{4}$ non compatibles avec NPV). Les résultats sont présentés en trois sous-sections (selon les prépositions de, à et pour).

\subsection{Avec la préposition de (NPV-de)}

Un premier sondage permet de repérer les $\mathrm{N}_{\mathrm{pc}}$ compatibles avec NPV-de (où PREP est instancié par la préposition de). Dans le tableau ci-dessous (comme dans le 3), on distingue les $\mathrm{N}_{\mathrm{pc}}$ bien attestés, à gauche, des $\mathrm{N}_{\mathrm{pc}}$ peu attestés, à droite :

Tableau 1. Les $\mathrm{N}_{\mathrm{pc}}$ compatibles avec NPV-de

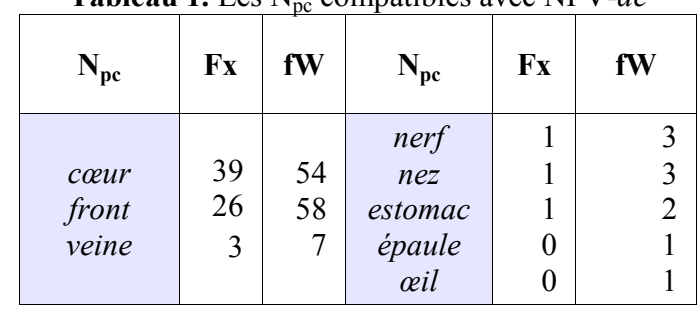

Commençons par les noms front, cour et veine les plus fréquents :

2. (a) je n'ai pas le cour de dire à ma mère que ma vie actuelle est celle d'une nomade (fW)

(b) Adamsberg avait eu le front d'exiger que les agents en roulements effectuent la garde éveillée (Fx)

(c) Grâce aux médias les prophètes religieux ont la veine d'avoir la parole (fW)

Pour ces trois noms, c'est l'article singulier qui est employé. Pour cœur et front, si on part du principe que le sens propre impose des contraintes aux sens figurés, cela peut sembler évident (l'être humain n'a qu'un cœur/front). Mais alors, il est étonnant que veine ne soit pas employé au pluriel (*les veines d'avoir la parole) comme cela est le cas pour d'autres effets de sens : la passion du théâtre coule dans * sa veine/ses veines.

Concernant ce dernier nom, on constate des attestations beaucoup moins nombreuses que celles de cour et front. En fait, le sondage met en évidence une concurrence avec une construction proche ( 8 occurrences dans Fx et 4 dans fW) dans laquelle le partitif est substitué à l'article : Vous avez de la veine d'être tombés sur nous $(\mathrm{Fx})^{5}$.

Pour cœur, il faut signaler un aspect que l'approche quantitative permet de mettre en évidence. Sur les 93 occurrences relevées, 79 (soit 84\%) sont à la forme négative (cf. 2a) et 9 expriment des interrogations directes (3a) ou indirectes (3b) :

3. (a) Qui aura le cour d'abandonner l'autre? (Fx)

(b) Il [...] lui demanda s'il aurait bien le cœur de tuer son Empereur (fW)

Cette nette proportion d'emplois à la forme négative ou dans des «contextes apparentés » (Muller, 2006 : 8-9) font de NPV-de employée avec cœur une expression à polarité négative au sens de Baker (1970).

Le tableau 1 indique encore trois noms (faiblement) attestés dans les deux bases :

4. (a) au moins les français ont-ils l'estomac de faire entendre leur voix (fW)

(b) ces pionniers capitalistes qui avaient le nez de miser très vite sur un nouveau produit (Fx)

(c) c'est à celui qui aura les nerfs de tenir jusqu'au bout (fW)

Les noms estomac et nez sont (logiquement) employés avec l'article le. Avec nerf, on pouvait s'attendre à l'emploi pluriel illustré en $(4 \mathrm{c})$ puisque c'est bien un réseau de nerfs qui 
parcourt le corps humain. Mais, il pourrait y avoir un flottement car on a trouvé : y aura donc pas un gas à poil qui ait le nerf de gueuler toutes ces vérités, nom de dieu ? (fW) ${ }^{6}$.

Terminons avec épaule et oeil (une seule occurrence dans fW) :

5. (a) Nasri risque de ne pas avoir les épaules de prendre le jeu à son compte à chaque match...

(b) tu as bien l'ail de remonter dans les archives comme ça

En fait, ces deux noms sont nettement plus fréquents avec NPV-pour. C'est pourquoi les commentaires les concernant sont regroupés à la section 2.3.

\subsection{Avec la préposition à (NPV-à)}

Confronté à la séquence avoir le cœur de, il est difficile de ne pas envisager la séquence alternative avoir le cour à. Plus loin, on tentera de saisir à quelle différence sémantique correspond cette différence syntaxique. Constatons simplement pour le moment que, à part cœur, seul un $\mathrm{N}_{\mathrm{pc}}$ entre dans la construction NPV-à : tête.

Tableau 2. Les $\mathrm{N}_{\mathrm{pc}}$ compatibles avec NPV-à

\begin{tabular}{|c|c|c|}
\hline $\mathbf{N}_{\mathbf{p c}}$ & $\mathbf{F x}$ & $\mathbf{f W}$ \\
\hline cœur & 41 & 84 \\
tête & 12 & 40 \\
\hline
\end{tabular}

Voici deux (des nombreux) exemples :

6. (a) J'ai même plus le cœur à écouter de la musique (Fx)

(b) Aujourd'hui, vois-tu, j'ai pas trop la tête à faire un post hyper intellectuel [...] (fW)

C'est la polarité négative des deux expressions qui attire l'attention. Sur les 125 occurrences de NPV-à avec cour, 114 sont à la forme négative (cf. 6a) ou relèvent de l'interrogation: ils pensent vraiment que nous avons le cœur à manger? (Fx). Picoche a déjà relevé cette singularité : " j'ai (ou, plus souvent, je n'ai pas) le cœur à rire » (1995 : 122, nous soulignons). Quant aux 52 occurrences de NPV-à avec tête, 48 sont à la forme négative, 3 à la forme interrogative - Croyez-vous que l'on a la tête à partir travailler quand on a son conjoint handicapé à la maison? (fW) - et seulement une à la forme affirmative (qui, en fait, renvoie au prix Reconnaissance québécois intitulé J'ai la tête à lire) ! Par conséquent, avoir le cour / la tête à sont assurément polarisés négativement ${ }^{7}$.

\subsection{Avec la préposition pour (NPV-pour)}

Plus loin (cf. 3.2.3), nous discuterons la pertinence de considérer NPV-pour comme une instanciation valide de NPV elle-même. On verra que la question mérite d'être posée tant le lien syntaxique instauré entre la locution avec $\mathrm{N}_{\mathrm{pc}}$ et le $\mathrm{V}_{\text {inf }}$ semble différent avec cette préposition. Pour le moment, contentons-nous d'un relevé d'occurrences :

Tableau 3. Les $\mathrm{N}_{\mathrm{pc}}$ compatibles avec NPV-pour

\begin{tabular}{|c|c|c|c|c|c|}
\hline $\mathbf{N}_{\mathbf{p c}}$ & $\mathbf{F x}$ & $\mathbf{f W}$ & $\mathbf{N}_{\mathbf{p c}}$ & $\mathbf{F x}$ & $\mathbf{f W}$ \\
\hline $\begin{array}{c}\text { épaule } \\
\text { ceil }\end{array}$ & 1 & 33 & $\begin{array}{c}\text { nerf } \\
\text { estomac } \\
\text { nez }\end{array}$ & 0 & 0 \\
0 & 19 & 2 \\
\hline
\end{tabular}

Avec épaule et $x i l$, la construction semble bien attestée dans fW :

7. (a) Bouchaud a les épaules pour porter le rôle-titre.

(b) Ah ben, il faut avoir l'oil pour choisir des lunettes sur le net... 
On note une différence significative d'attestations entre les deux bases de données. Sans doute, NPV-pour relève-t-elle davantage d'un usage courant. Pour épaule, le nom est employé au pluriel en cohérence avec le fait que la partie du corps en jeu est, comme bien d'autres, double (un emploi singulier est exclu : *Bouchaud a l'épaule pour le rôle-titre).

Par contre, le nom oeil est au singulier dans NPV malgré son référent corporel double (les yeux). De fait, les rares occurrences de la séquence les yeux pour $V_{\text {inf }}$ relèvent précisément d'un emploi propre : alors qu'elle était si proche d'eux, s'ils avaient les yeux pour la voir (fW). Cela dit, il faut noter que, même avec oeil (singulier), NPV tend à exploiter un sens peu éloigné du sens physiologique (cf. $7 \mathrm{~b}$ ) comme en témoignent les $\mathrm{V}_{\text {inf }}$ (voir, distinguer, repérer...) qui suivent la préposition.

Les noms nerf et estomac présentent seulement quelques occurrences dans fW :

8. (a) Faut tout de même avoir les nerfs pour travailler dans une hotline ;-)

(b) je me doutais que vous n'aviez pas l'estomac pour tenir un blog sur la Coupe du Monde avec le ton adopté au départ

Une comparaison avec le tableau 1 pousse à considérer que ces noms sont peu employés avec NPV et que l'usage hésite entre les prépositions de et pour. Dans les énoncés trouvés ici, nerf est toujours employé au pluriel (l'emploi singulier signalé à la section 2.1 s'en trouve marginalisé - cf. la note 6 cependant) et estomac au singulier.

Terminons en commentant le seul énoncé avec nez trouvé sur fW :

9. Je suis surtout sensible à ce petit quelque chose de poudré fait, paraît-il (mais je n'ai pas le nez pour distinguer tout ça), de violette, de bergamote, de cèdre, de musc et de pivoine

Dans un tel énoncé, le nom nez conserve un sens corporel (il s'agit bien de la partie du corps qui permet de sentir) et ne relève pas d'un emploi figuré. On peut difficilement considérer cet exemple comme une - unique qui plus est - occurrence de NPV.

\subsection{Bilan}

On retiendra la compatibilité de coeur, front et veine avec NPV-de, de cœur et tête avec NPV-à et, malgré la nette disproportion d'attestations entre Fx et fW, de épaule et oil avec NPV-pour. Pour nez, nous considérons qu'il relève de NPV-de. Enfin, estomac et nerf sont considérés comme également compatibles avec NPV-de et NPV-pour. Il ne sera tenu compte que de façon anecdotique des attestations de NPV-de avec épaule et ceil. Car, d'une part, elles sont réduites à une seule occurrence et, d'autre part, la compatibilité avec NPVpour apparait comme une alternative bien plus convaincante.

\section{Analyse sémantique}

Sur le plan sémantique, les données invitent à distinguer deux perspectives : l'une sur la construction NPV elle-même (3.2) et l'autre sur chacun des $\mathrm{N}_{\mathrm{pc}}$ compatibles avec NPV (3.3). Auparavant, une distinction entre deux acceptions des $\mathrm{N}_{\mathrm{pc}}$ doit être explicitée (3.1).

\subsection{Distinction entre les emplois PART et QUAL des $\mathbf{N}_{\mathrm{pc}}$}

Dans notre thèse (2018), est proposé un panorama de la variation sémantique des $\mathrm{N}_{\mathrm{pc}}$. Nous reprenons ici les éléments utiles à l'étude de NPV en partant de la distinction aristotélicienne entre nécessité et contingence (cf. Gosselin et François, 1991).

Si on considère le sens réputé premier d'un nom comme nez, on peut considérer que la propriété avoir un nez est nécessaire sur le plan référentiel (i.e. tous les êtres humains ont un $n e z^{8}$ ) comme sur le plan temporel (i.e. à tout moment, les êtres humains ont leur nez). La partie du corps humain est donc une partie nécessaire ${ }^{9}$. 
Cette première observation appelle trois commentaires. Premièrement, on retrouve ce caractère nécessaire dans des emplois relevant de la catachrèse : les pieds sont nécessaires à la table ${ }^{10}$ (les pieds de la table) comme le nez à l'avion (le nez de l'avion).

Deuxièmement, cette nécessité se traduit syntaxiquement par une forte compatibilité avec les structures de possession inaliénable (Van Peteghem, 2006) du type Paul a le nez écorché / cette table a les pieds cassés ou Paul conduit les yeux fermés / l'avion monte dans le ciel le nez vers le haut.

Troisièmement, ce caractère nécessaire n'est en rien remis en cause par des emplois figurés où le $\mathrm{N}_{\mathrm{pc}}$ ne réfère plus à une partie du corps humain (au sens physiologique). Les exemples sont nombreux :

10. Colin a le nez creux / le cour en peine / le dos large / la tête dure

Ainsi, tous les emplois de nez évoqués précédemment (Colin a le nez écorché, le nez de l'avion, Colin a le nez creux) relèvent d'une acception partie nécessaire (PART) en dépit de la pluralité référentielle (corps humain, objet, psychisme).

À la nécessité, Aristote oppose la contingence. Par exemple, la propriété avoir de l'audace est contingente sur le plan référentiel (i.e. certains êtres humains sont audacieux, d'autres non) comme sur le plan temporel (i.e. un être humain peut être audacieux à certains moments, pas à d'autres). Dans sa Rhétorique, Aristote appelle habitus les « vertus et les vices » qui correspondent aux « tendances de chacun » (XII, 1388b). En linguistique, on parle généralement de qualité ${ }^{11}$. Or, dans certains emplois, un $\mathrm{N}_{\mathrm{pc}}$ peut relever de la qualité (QUAL) :

11. (a) Colin a du nez [Colin a de l'intuition $\left.{ }^{12}\right]$

(b) Colin a du ventre [Colin a du courage]

(c) Colin a du cœur ${ }^{13}$ [Colin est généreux]

Contrairement à ce que ces exemples prototypiques semblent indiquer (et qui occuperont ici notre attention), la qualité ne relève pas forcément du psychologique. Un énoncé comme Paul a du ventre peut se comprendre comme une description physiologique (cf. Paul a du bide). De même, Paul a des jambes renvoie davantage à une aptitude physique. Dans certains cas, la frontière entre psychologique et physiologique est floutée : ainsi, dans Paul a de l'oreille, le nom oreille renvoie autant à un «sens musical » (psychocognitif) qu'à une "aptitude perceptive » (physiologique). Par ailleurs, les emplois de ce type ne sont pas réservés à la sphère humaine - cf. Bertin (2018: 212). Les exemples sont certes plus rares mais bien présents dans l'usage de certains locuteurs : ce vin a de la cuisse, ce mur a du pied [une bonne largeur de base], ce papier a de la main [de la tenue].

Comme le suggère Hanon (1988: 164), la portée de la négation oppose nettement la structure phrastique de possession inaliénable qui correspond à la partie nécessaire et l'emploi absolu (avec partitif) qui correspond à la qualité :
12. (a) Paul n'a pas le cœur brisé
[Paul a un cœur mais ce cœur n'est pas brisé]
(b) Paul n'a pas de cœur
[Paul n'a pas la qualité qui correspond au cœur]

\subsection{Contribution sémantique du $\mathrm{N}_{\mathrm{pc}}$ dans NPV}

\subsubsection{La construction NPV-de}

Les noms cœur, veine, estomac et nez manifestent une régularité intéressante. En effet, à chaque emploi absolu (qualité psychologique), correspond un emploi de NPV-de :

13. (a) Colin a du cœur

(b) Colin a de la veine

(c) Paul a de l'estomac

(d) Paul a du nez
Colin n'a pas le cœur de dire la vérité

Colin a eu la veine de partir à temps

Paul a eu l'estomac de s'exprimer

Paul a le nez d'aller avec eux [la force / l'énergie]

[la chance]

[le courage]

[l'intuition] 
Syntaxiquement, ces emplois sont tout à fait équivalents à des emplois du type Colin $a$ la gentillesse / le courage de dire la vérité ${ }^{14}$. Dans ces séquences isomorphes à NPV-de, c'est bien un nom de qualité qui remplace le $\mathrm{N}_{\mathrm{pc}}$.

Ce premier constat appelle deux commentaires. D'abord, avec cœur, on observe que la construction NPV-de active plutôt, en concurrence avec estomac, l'effet de sens 'courage' (le cœur à l'ouvrage) plutôt que l'effet de sens 'générosité' (avoir bon cœur). Ensuite, avec veine, la construction alternative repérée plus haut (Colin a eu de la veine de partir à temps) qui amalgame NPV elle-même et l'emploi absolu (avec partitif) ${ }^{15}$ ne fait finalement que confirmer le lien sémantique entre NPV et qualité.

Avec nerf, on retrouve le fonctionnement illustré en (13) : à l'emploi absolu où le nom évoque une qualité (la vigueur pour le Petit Robert 2014, PR), correspond un emploi QUAL dans NPV-de. Le syntagme est souvent au pluriel (les nerfs) mais les rares emplois singuliers (le nerf) pourraient se construire en référence à l'emploi absolu (du nerf) :

14. Colin a du nerf Colin a eu le/s nerf/s de tenir jusqu'au bout [la vigueur]

Le nom front est plus problématique car il ne donne pas lieu à un emploi absolu (?Paul $a d u$ front $^{16}$ ) qui relèverait de la qualité. Malgré cette lacune distributionnelle, les gloses tendent bien à confirmer un emploi QUAL (psychologique) au sein de NPV-de :

15. * Colin a du front Colin a le front de venir à cette réunion [l'audace]

On a vu (cf. note 12) qu'un nom de qualité ne donne pas toujours lieu à un emploi absolu (? Paul a de la gentillesse). L'absence de correspondance avec un emploi absolu n'interdit donc pas l'interprétation QUAL de front dans NPV-de. On s'en tiendra donc à cette interprétation qui nous parait la plus cohérente.

Rappelons en outre que, pour Aristote, « l'habitus [la qualité] est aussi considéré comme ce qui fonde la possibilité d'un acte, ou d'une passion » (Gosselin \& François, 1991 : 71) ${ }^{17}$. Or, la construction NPV, en combinant un $\mathrm{V}_{\text {inf }}$ (préférablement agentif et dynamique) avec un $\mathrm{N}_{\mathrm{pc}}$, évoque une action (cf. dire, partir, aller, s'exprimer dans les exemples précédents) mise en œuvre par la mobilisation d'une qualité précisément exprimée par ce nom.

En conclusion, dans la construction NPV-de, l'emploi du $\mathrm{N}_{\mathrm{pc}}$ relève de la qualité. En cohérence avec le principe d'un emploi figuré du $\mathrm{N}_{\mathrm{pc}}$, cette qualité est toujours d'ordre psychologique.

\subsubsection{La construction NPV-à}

On l'a vu, seuls les noms cœur et tête sont compatibles avec la construction NPV-à (Colin n'a pas la tête à travailler / le cour à rire). Cette construction alternative invite à s'interroger sur l'éventuelle différence sémantique qu'elle reflète.

On partira du constat que, dans la construction en $\grave{a}$, ce n'est pas un nom de qualité (QUAL) qui peut être substitué au $\mathrm{N}_{\mathrm{pc}}$ mais plutôt un nom de partie nécessaire (PART) comme esprit ou âme (Mostrov, $2015: 142$ ) :

16. (a) Colin n'a pas la tête / *l'intelligence / l'esprit à travailler

(b) Colin n'a pas le cœur / le * courage / l'âme à rire

Ce contraste suggère que, d'un point de vue sémantique, les emplois de tête et cœur dans la construction NPV-à relèvent de la partie nécessaire et non de la qualité. Même si on a vu (avec front) que ce critère avait ces limites, le fait que, à la différence de cour, tête n'admette pas d'emploi QUAL ( ${ }^{*}$ Paul a de la tête ${ }^{18}$ ) va dans le sens de cette interprétation.

Pour confirmer cette différence sémantique dans les emplois (PART plutôt que QUAL), deux paramètres complémentaires méritent d'être étudiés. Le premier est l'aspect grammatical du verbe avoir et le second l'aspect lexical de $\mathrm{V}_{\text {inf. }}$ Pour ce qui concerne le premier paramètre, partons des contrastes suivants :

17. (a) hier, Colin ?n'a pas eu / n'avait pas la tête / le cœur à travailler

(b) hier, Colin n'a pas eu / ?n'avait pas le cœur de travailler 
Avec NPV-à, le passé composé semble moins naturel que l'imparfait alors que c'est le contraire avec NPV-de. Comme cette différence peut sembler ténue, nous avons cherché à l'éprouver dans Fx et fW :

Tableau 4. Flexions de avoir dans NPV-à et NPV-de dans Fx et fW

\begin{tabular}{|c|c|c|c|}
\hline & imparfait & p. composé & autres \\
\hline avoir le coeur de & 12 & 27 & 54 \\
\hline $\begin{array}{c}\text { avoir le cour à } \\
\text { avoir la tête } \grave{a}\end{array}$ & 38 & 4 & 73 \\
\hline
\end{tabular}

Le contraste est net avec avoir la tête à. Le contraste moins probant avec cour (qui semble plutôt relever d'une tendance) pourrait être dû au fait que, les deux constructions étant précisément possibles, les locuteurs les amalgament et ne font plus toujours une différence bien nette.

Cette différence suggère que la construction en à privilégie une présentation non bornée du procès (qui dure et « s'étale » sans limites) alors que la construction en de appelle plutôt un procès borné ${ }^{19}$. On retrouve un tel contraste au présent :

18. en ce moment, Paul n'a pas le cœur à/?de rire / la tête à travailler

Corollairement, NPV-à tolère mal l'introduction d'un verbe à l'infinitif exprimant un aspect lexical perfectif alors que c'est tout fait possible avec NPV-de :

19. (a) Paul n'a pas le cœur de lui ouvrir la porte / terminer ce devoir / se lever

(b) ?Paul n'a pas le cœur à lui ouvrir la porte / terminer ce devoir / se lever

(c) Paul n'a pas le cœur à travailler / rire

Notons, pour terminer, que, dans les bases de données, avec NPV-à, on constate une surreprésentation de prédicats du type faire de l'humour / la fête, célébrer et rire (en fait, avoir le cour à rire semble relever de l'idiomatique).

Ces éléments suggèrent que, avec NPV-à, ce qui est en jeu, c'est un certain état dans lequel se trouve la partie nécessaire (ici, une partie du corps envisagée comme relevant du psychologique) désignée par cœur ou tête : la personne est - ou, plus sûrement, n'est pas dans une "certaine disposition » vis-à-vis d'une certaine activité. Par contraste, et en cohérence avec ce qui a été dit à la section précédente, avec NPV-de, l'emploi du nom cœur permet d'instancier une qualité mise en œuvre pour prendre en charge un procès-action.

Ces observations font écho à l'analyse que développe Cadiot sur les prépositions $a ̀$ et $d e$ suivis de "compléments infinitivaux ». En effet, pour cet auteur, "à sert à marquer une saisie du sens du complément à l'infinitif à partir du sujet, alors que de nous situe dans une phase neutre entre le sujet et l'objet, dans le simple prolongement du prédicat » (1993: $94)^{20}$. De fait, avec NPV-à, l'action à laquelle réfère le verbe à l'infinitif, seulement potentielle, est relative à une disposition caractérisant le sujet alors que, avec NPV-de, ce qui est mis en avant, c'est un procès effectif ${ }^{21}$.

\subsubsection{La construction NPV-pour}

Comme l'illustrent les énoncés suivants, les compléments introduits par de et pour ont manifestement des statuts syntaxiques différents :

20. (a) Colin a le front de s'opposer au patron / ??de s'opposer au patron, Colin a le front

(b) Colin a les épaules pour s'opposer au patron / pour s'opposer au patron, Colin a les épaules

Le test de dislocation confirme la plus grande autonomie du complément en pour :

21. (a) ??le front, Colin l'a de s'opposer au patron

(b) les épaules, Colin les a pour s'opposer au patron 
En fait, la différence vaut aussi avec $\grave{a}$ et pour et n'est pas propre à NPV. Ainsi, opposant ??à / pour mon anniversaire, Pierre est venu, Anscombre observe que "pour opère une discontinuité entre les deux procès $P_{1}$ et $P_{2}$ qu'il relie » $(1991$ : 34). Pourtant, il serait abusif d'envisager le complément en pour en (20b) comme circonstanciel.

En effet, Colinet et al. (2014) donnent des critères permettant de distinguer un « complément sous-catégorisé » d'une "subordonnée adverbiale infinitive ». Deux de ces critères ont été évoqués plus haut: la difficulté de transformer l'infinitive en une subordonnée en pour que (cf. le test de "périphrase verbale » évoqué dans notre propos liminaire) et la possibilité de substituer de à pour (cf. le flottement observé entre avoir les nerfs/l'estomac de et avoir les nerfs/l'estomac pour - moins probant, il est vrai avec épaules et $\propto i l)$. Par ailleurs, « lorsque la préposition [pour] est sous-catégorisée, elle peut apparaitre seule, c'est à dire sans son objet » (2014: 3049). Or c'est ici le cas :

22. (a) Colin pourra-t-il faire face à ça ? Oui, il a les épaules pour / l'œil pour

(b) la fille qui occupait la présidence de celle-ci n'avait en aucun cas les épaules pour (fW)

Enfin, quand deux compléments en pour sont co-occurrents, l'ordre est contraint, le premier est sous-catégorisé et le second est adverbial (2014:3050) :

23. (a) Colin a les épaules pour s'opposer au patron, pour obtenir une augmentation

(b) ??Colin a les épaules pour obtenir une augmentation, pour s'opposer au patron

Ces observations de niveau syntaxique confirment que NPV-pour est une construction en tant que telle. Sémantiquement, on retrouve un fonctionnement similaire à NPV-de :

23. (a) Paul a les épaules pour affronter le patron [la force]

(b) Paul a les nerfs pour travailler dans une hotline [la vigueur]

(c) Paul n'a pas l'estomac pour s'opposer à son chef [le courage]

Dans tous ces énoncés, le $\mathrm{N}_{\mathrm{pc}}$ renvoie, dans un sens figuré, à une qualité psychologique. Dans les cas de nerf et estomac, c'est en fait la même qu'avec NPV-de - cf. les énoncés (14) et (13c). Si l'utilisation de la préposition pour (à la place de de) donne plus d'autonomie au complément, elle ne modifie donc pas l'interprétation sémantique du $\mathrm{N}_{\mathrm{pc}}$.

Avec $\propto i l$, c'est plus délicat: on ne voit pas vraiment à quelle qualité psychologique l'instanciation de ce nom ferait appel (en tout cas, nous ne voyons pas quel lexème l'exprimerait). D'ailleurs (cf. 1.3), pour est le plus souvent suivie d'un verbe de perception :

24. (a) c'est tout bête comme cliché, mais faut avoir l'œil pour le voir, j'adore (fW)

(b) là aussi, il faut avoir l'œil pour retrouver son chemin (fW)

(c) elles ont l'œil pour te détecter le pisseur à vingt balles [...] (Fx)

En outre, en dépit de l'égale inacceptabilité de *Paul a des épaules/de l'œill ${ }^{22}$, nous interprétons, en tant que locuteur francophone, beaucoup plus facilement l'énoncé (5a) (Nasri risque de ne pas avoir les épaules de prendre le jeu à son compte [...]) que l'énoncé (5b) (tu as bien l'œil de remonter dans les archives comme ça).

En résumé, les indices sémantiques témoignent d'un apparentement de NPV-pour avec NPV-de et lui confèrent un statut de "construction alternative » dans laquelle des noms comme estomac, nerf et surtout épaule (nous excluons désormais $a i l$ ) renvoient à une qualité psychologique (QUAL).

\subsection{Vers une sémantique des $\mathbf{N}_{\mathrm{pc}}$}

Dans une logique programmatique, nous nous appuyons sur les éléments d'analyse précédents pour identifier en quoi la compatibilité des $\mathrm{N}_{\mathrm{pc}}$ avec NPV donne des pistes pour analyser sémantiquement ces noms. 


\subsubsection{Les noms tête, cœur (et estomac)}

Au-delà du fait que tête et cœur sont parmi les $\mathrm{N}_{\mathrm{pc}}$ les plus polysémiques, leur rapprochement est motivé par leur commune compatibilité avec NPV-à. Par ailleurs, on l'a $\mathrm{vu}$, ces noms semblent imposer à NPV-à une polarité négative. Cette polarité pourrait évidemment venir de la construction elle-même (cf. note 7) mais, comme - contrairement à ce que nous affirmions dans Bertin (2018: 188) - cœur semble également imposer une polarité négative à NPV-de (à la différence de front par exemple), il semble raisonnable de penser que les noms tête et cœur jouent un rôle crucial dans ce phénomène.

Dans un article de synthèse consacré à cœur (2019), nous avons pointé la valuation fortement positive associée à cœur qui renvoie à un organe vital, une partie psychologique cruciale (cf. briser le cœur) marquée d'une modalité appréciative positive (avoir bon cour / du cœur, de bon cœur...) comme une zone de quintessence d'un domaine (cf. cour de la forêt, cour du problème). Or, le nom tête qui renvoie aussi à une partie du corps envisagée comme vitale (au point que tête est synonyme de vie dans certains emplois : sa tête est mise à prix) pourrait intégrer une dimension comparable dans sa signification lexicale.

De ce point de vue, la polarité négative que les deux noms imposent à NPV constitue peut-être un indice. On a vu que NPV-de privilégiait un $\mathrm{V}_{\text {inf }}$ exprimant un procès agentif (cf. 3.2.1). C'est sans doute moins vrai avec NPV-à (cf. Paul n'a pas le cour à être heureux). En revanche, en plus d'une polarité négative, on observe que, avec cœur $(\grave{a} / d e)$ et tête $(\grave{a})$, c'est un procès « désirable ${ }^{23}$ » qui est privilégié :

25. (a) Paul n'a pas le cœur à être heureux / le cœur de travailler /la tête à rire

(b) ?Paul n'a pas le cœur à être malheureux / le cœur de s'épuiser au travail / la tête à pleurer

$\mathrm{Au}$ fond, ce que semblent imposer cœur et tête, c'est une polarité négative qui s'articule à l'instanciation d'un procès marqué par une modalité appréciative positive (ou, du moins, non négative). D'un point de vue énonciatif, on explicite ainsi l'absence d'une disposition ou d'un enthousiasme pour la mise en œuvre d'un procès intrinsèquement désirable (rire, de façon prototypique).

Il resterait à comprendre ce qui distingue cœur de tête (dans de tels énoncés). La compatibilité différente avec NPV-de fournit une piste. On a vu que la compatibilité de cœur avec NPV-de indique que son référent (psychologique) est envisagé comme «moteur» qui porte à l'action (QUAL) quand l'incompatibilité de tête avec NPV-de cantonne son référent à une simple «disposition» relevant d'un certain état (PART). Or, cette différence de "dynamicité » est congruente avec les emplois propres de cœur et tête: le cœur est bien le moteur qui permet au corps de se mouvoir quand la tête renvoie davantage à l'intellect et au cérébral.

Pour estomac, on a vu que, en complément de son emploi absolu (cf. avoir de l'estomac), sa compatibilité avec NPV-de et NPV-pour donnait lieu à l'expression d'une qualité relevant du courage (le $P R$ donne comme synonymes courage et, précisément, cœur). Cependant, le dictionnaire qualifie également cet emploi de «vieux » expliquant ainsi la faible quantité d'occurrences relevées dans les bases de données. Il semble que le nom ait perdu, au profit de $c œ u r^{24}$, sa place de $\mathrm{N}_{\mathrm{pc}}$ pouvant évoquer le courage.

\subsubsection{Les noms front, épaule (et dos)}

Les noms front et épaule ont en commun d'être compatibles avec NPV sans admettre un emploi absolu relevant de la qualité (Colin a du *front / des *épaules). Mais surtout, les deux noms instanciés au sein de NPV donnent lieu à des interprétations très similaires (pas sans rapport, il est vrai, avec celles de cour ou estomac) :

27. je ne sais pas si Colin aura le front de / les épaules pour affronter le patron 
Dans les deux cas, il semble qu'on évoque un mélange d'audace et de courage orienté vers une action à accomplir. Pour épaule, nous avons proposé, dans un récent article (sous presse), de le rapprocher de dos sur la base du fait que ces deux noms renvoyaient à l'idée d'une partie du corps susceptible de porter une charge (Paul porte Colin sur son dos / ses épaules mais aussi, dans un sens plus figuré, Paul courbe le dos / les épaules).

Or, on peut montrer, dans le cas de dos, que le charge est généralement subie (cf. Paul en a plein le dos, Colin a son patron sur le dos, Matthieu lui a mis cette responsabilité sur le dos) alors que, avec épaule, il s'agit plutôt d'une force positive (Matthieu a désormais cette responsabilité sur les épaules, Colin a la tête sur les épaules). Dès lors, il est significatif que épaule, et non dos, soit compatible avec NPV :

28. Madame Ségolène n'a pas les épaules [*le dos] pour gouverner la France (fW)

Pour ce qui concerne front, pour lequel nous n'avons pas encore développé d'étude spécifique, une autre acception de ce nom indique une piste à suivre. En effet, à l'instar de pied ou cour notamment, front donne également lieu à un sens positionnel : la maison est située sur le front de mer, des banderoles ont été accrochées au front du bâtiment. Ce sens positionnel est même fortement lexicalisé dans le syntagme le front où il renvoie sans autre précision co-textuelle à la " zone active d'un conflit armé » (cf. partir / mourir au front).

La valeur de position avancée (voire exposée) qu'activent ces emplois met en lumière une piste d'analyse pour comprendre, en lien avec son sens dans NPV, la signification lexicale de front. En effet, avoir le front de faire quelque chose, c'est aller «avec résolution » au-devant d'ennuis potentiels (cf. les dérivés affronter et confronter).

\subsubsection{Les noms nerf, veine (et artère)}

Il est tentant de rapprocher nerf et veine sur la base de leurs sens propres respectifs. Tous deux réfèrent en effet à des " conduits » organisés en réseaux irriguant tout le corps. Par ailleurs, les noms nerf et veine sont tous deux compatibles avec NPV-de et y renvoient à une qualité (avoir du nerf / de la vigueur; avoir de la veine / de la chance).

On a vu cependant que, morphologiquement, les usages différaient au moins sur un point. Quand nerf s'emploie généralement au pluriel dans NPV (malgré avoir du nerf), le nom veine s'emploie nécessairement au singulier (avoir *les veines de $V_{i n f}$ ).

En fait, sur le plan sémantique, certains éléments sont à expliciter qui montrent que les potentialités sémantiques de nerf et veine sont assez différentes. Ainsi, pour nerf, si les énoncés (4c) et (8a) repris ci-dessous confirment l'expression d'une qualité (la vigueur),

4. (c) c'est à celui qui aura les nerfs de tenir jusqu'au bout (fW)

8. (a) Faut tout de même avoir les nerfs pour travailler dans une hotline ;-)

c'est beaucoup plus discutable pour l'énoncé suivant :

29. Y'a ceux qui n'ont pas de probléme qui veulent bien commenter, les autres ont vraiment trop les nerfs pour lire cette note (fW)

Cet énoncé ne se glose certainement pas « les autres ont trop de vigueur pour lire cette note » mais plus sûrement « les autres sont trop agacés pour lire cette note ». Dans cette séquence, l'insertion du syntagme adverbial vraiment trop conduit à faire émerger l'idée d'un état plutôt que d'une qualité. Cette observation est congruente avec l'identification d'une acception ETAT pour certains $\mathrm{N}_{\mathrm{pc}}$ dont nerf précisément (cf. Bertin, 2018 : 189) :

30. Paul a les foies [peur] / glandes [dégoût] / les nerfs [agacement]

Ainsi, l'analyse sémantique de nerf devrait tenir compte de cette distinction dans les emplois. S'il est probable que la qualité et l'état ne sont pas sans lien (la vigueur comme l'énervement peuvent s'interpréter comme des traductions différentes d'une certaine énergie qui émane du sujet), il n'en reste pas moins qu'il s'agit bien de deux acception différentes. 
Le nom veine n'a pas d'emplois ETAT. Ce qui ne l'empêche pas de receler un grand potentiel sémantique. En effet, à côté des emplois déjà identifiés, on trouve un emploi «PART-psychologique » (a), un emploi Objet (b) et un emploi plus difficile à classer (c) :

31. (a) Malvina se tenait droite, comme si la toque, telle la mitre d'un évêque, lui avait infusé dans les veines la conviction d'être au-dessus de toutes les vicissitudes de l'existence (Fx)

(b) on trouve aussi dans le même endroit une petite veine de sable (fW)

(c) le chapitre des Tchétchènes est dans la veine des précédents (Fx)

L'emploi en (31b) rappelle certains emplois du nom artère (cf. l'artère principale $d u$ centre-ville) qui renvoient à une voie de grand passage même si veine désigne plutôt un conduit étroit ou un « filon mince » $(P R)$. En fait, le rapprochement s'arrête là car artère n'a pas d'emplois QUAL (*Paul/cette ville a de l'artère). Ainsi, la polysémie de veine se révèle assez singulière, d'autant que le rapport entre les différents sens n'est pas très immédiat.

Il faut par ailleurs ajouter que la qualité exprimée par veine en emploi absolu (avoir de la veine / chance i.e. être chanceux) comme dans NPV (avoir la veine / chance de) présente un profil particulier. En effet, comme l'illustrent les contrastes suivants, la notion de chance renvoie à une qualité moins « agentive » que le courage ou la générosité par exemple :

32. (a) Colin a fait preuve de courage / générosité / ?chance

(b) Colin n'a pas le ??cœur / ?front / la veine d'être en pleine forme / d'avoir un vélo

(c) Colin n'a pas eu le ??cœur / ?front / la veine d'être ramené chez lui

En (32a), le prédicat faire preuve de (cf. note 11) sélectionne une qualité dont Colin est à l'initiative mais cela est moins probant avec chance. À l'inverse, en (32b), le caractère statif des prédicats être en pleine forme ou avoir un vélo (cf. également $2 \mathrm{c}$ ) s'accommode mieux de veine que de cour ou front (dans NPV). De même, en (32c), la forme passive qui impose un rôle de patient à Colin est plus envisageable avec veine qu'avec cœur ou front.

Ainsi, même au sein du paradigme des $\mathrm{N}_{\mathrm{pc}}$ compatibles avec NPV-de, le nom veine manifeste des propriétés sémantiques un peu spécifiques qu'il reste à mieux établir en lien avec ses autres effets de sens.

\subsubsection{Les noms nez, œil (ainsi que oreille et bouche)}

Les noms nez, ceil, oreille et bouche seront abordés rapidement pour constater que, si ces quatre $\mathrm{N}_{\mathrm{pc}}$ présentent une cohérence sémantique de surface (ils désignent tous une partie de la tête bien identifiée), les constructions avec lesquelles ils sont compatibles et les acceptions auxquelles ils donnent lieu (pour s'en tenir aux observations formulées dans le cadre de cet article) sont finalement assez différentes.

Quand les noms nez et oreille donnent tous les deux lieu à un emploi absolu pour évoquer une qualité à la frontière du psychologique et du physiologique (Colin a du nez / de l'oreille), de tels emplois semblent plus difficiles avec oeil et bouche. En fait, le $P R$ donne bien avoir de l'œil dans le sens « avoir un bel aspect » (qualité proprement physiologique) mais il précise que l'emploi est vieilli (aucune attestation dans Fx et fW). Par ailleurs, l'expression avoir de la bouche (là encore, peu usitée) semble réservée à un sujet non humain (ce vin a de la bouche).

Quant à la compatibilité avec NPV, elle ne présente pas le caractère régulier qu'on aurait pu espérer. Si la construction est compatible avec nez (Paul a eu le nez de venir), elle ne l'est pas avec oreille (*Paul a eu l'oreille de venir). Et si, malgré les réticences explicitées plus haut, on considère qu'elle l'est avec ail (Paul a l'œil pour dénicher de bonnes affaires), c'est sans rapport avec le sens de avoir de l'œil (de toute façon litigieux). Enfin, le nom bouche est incompatible avec NPV (*avoir la bouche de).

Ces premières observations justifient des études séparées même si elles peuvent éventuellement être «contrastées » (à l'instar des couples dos/épaule et artère/veine) sur la base d'une propriété commune (ici, la référence à un nom de partie de la tête) dont il resterait à prouver le caractère crucial d'un point de vue sémantique. 


\section{Conclusion}

Nous avons étudié NPV, une construction mettant en jeu un «nom de partie du corps humain ». Pour cela, nous avons d'abord repéré les $\mathrm{N}_{\mathrm{pc}}$ concernés (cœur, front, veine, tête, épaule et, dans une moindre mesure, estomac, nerf, nez plus oeil finalement rejeté). Nous avons ensuite essayé de comprendre le rôle sémantique du $\mathrm{N}_{\mathrm{pc}}$ dans NPV. Cela a conduit à distinguer un emploi QUAL dans NPV-de et un emploi PART dans NPV-à. Dans NPVpour, envisagée comme une "construction » alternative à NPV-de, l'emploi du $\mathrm{N}_{\mathrm{pc}}$ relève également d'une qualité mobilisée pour mettre en œuvre une action. Enfin, de la compatibilité avec NPV, nous avons cherché à tirer des conséquences pour l'analyse sémantique des $\mathrm{N}_{\mathrm{pc}}$ en jeu. Sans reprendre tous les éléments, on peut souligner une diversité de fonctionnements syntaxico-sémantiques. Comme on l'a notamment constaté, la référence corporelle (relevant du «sens propre» de chaque $\mathrm{Npc}$ ) n'est pas un critère très probant d'unité. Ainsi, NPV-à est compatible avec un nom d'organe (cœeur) et un nom de partie du corps externe (tête). Corollairement, les principaux noms de parties de la tête (bouche, nez, ceil, oreille) ont des compatibilités très différentes avec NPV suggérant des modes de variation de sens très différents. Nous sommes conscient que cette étude mériterait d'être complétée. Ainsi, focalisé sur le sens du $\mathrm{N}_{\mathrm{pc}}$, nous n'avons fait qu'effleurer la contribution sémantique de la préposition dans NPV qui semble jouer un rôle déterminant dans le mode d'instanciation du procès auquel réfère $V_{\text {inf. }}$ Par ailleurs, nous avons évoqué le statut de périphrase verbale de NPV. Or il resterait à étudier la valeur modale de cette périphrase verbale de même qu'on étudie la valeur aspectuelle de certaines autres (cf. venir de).

\section{Références bibliographiques}

Anscombre, J.-C. (1991). L'article zéro sous préposition. Langue française, 91, 24-39.

Anscombre, J.-C. (1995). Morphologie et représentation événementielle : le cas des noms de sentiment et d'attitude. Langue française, 105, 40-54.

Ansombre, J.-C. (2001). Le rôle du lexique dans la théorie des stéréotypes. Langages, 142, 57-76.

Aristote (1991). Rhétorique. Paris : Le livre de poche.

Baker, C.L. (1970). Double negatives. Linguistic Inquiry, 1, 169-186.

Bertin, T. (2018). La polysémie des noms de parties du corps humain en français - Analyse sémantique de artère, bouche, cœur, épaule et pied. Thèse soutenue à l'université de Rouen.

Bertin, T. (2019). De l'invariance du cœur. L'information grammaticale, 162, 27-33.

Bertin, T. (sous presse). Les noms dos et épaule - Signification lexicale, référence et dénomination.

Bouveret, M. et Sweetser, E. (2009). Multi-frame semantics, metaphoric extensions and grammar, Proceedings of the 35th Annual Meeting of the Berkeley Linguistics Society [En ligne] Consulte le 4 mai 2020. URL : http://linguistics.berkeley.edu/ sweetser/Bouveret.SweetserBLS09.pdf

Cadiot, P. (1993) De et deux de ses concurrents : avec et à. Langages, 110, 68-106.

Chappell, H. et McGregor, W. (1996) The grammar of inalienability - A typological perspective on body part terms and the part-whole relation. Berlin \& New York : Mouton de Gruyter.

Colinet, M., Danlos, L., Dargnat, M. et Winterstein, G. (2014). Emplois de la préposition pour suivie d'une infinitive: description, critères formels et annotation en corpus. Actes du Congrès de Linguistique française - SHS Web of Conferences, 8, 3041-3058.

Dingemanse, M. (2009). The selective advantage of body-part terms. Journal of Pragmatics, 41, 2130-2136.

Flaux, N. et Van de Velde, D. (2000). Les noms en français : esquisse de classement. Gap : Ophrys.

Goossens, V. (2005). Les noms de sentiment : Esquisse de typologie sémantique fondée sur les collocations verbales. Lidil, 32, 103-121.

Gosselin, L. et François, J. (1991). Les typologies de procès : des verbes aux prédications. Travaux de linguistique et de philologie, 29, 19-86. 
Gosselin, L. (1996). Le traitement de la polysémie contextuelle dans le calcul sémantique. Intellectica, 22-1, 93-117.

Gosselin, L. (2015). De l'opposition modus / dictum à la distinction entre modalités extrinsèques et modalités intrinsèques. Bulletin de la société linguistique de Paris, $C X-1,1-50$.

Gosselin, L. (sous presse). Aspect et formes verbales en français.

Gross, M. (1999). Sur la définition d'auxiliaire du verbe. Langages, 135, 8-21.

Hanon, S. (1988). Qui à quoi ? Réflexions sur la possession inaliénable et le verbe avoir en français. Revue romane, 23, 161-177.

Kleiber G. (1999). Problèmes de sémantique : la polysémie en questions. Villeneuve d'Ascq : Presses universitaires du Septentrion.

Kraska-Szlenk, I. (2014). Semantic extensions of body part terms: common patterns and their interpretation. Language Sciences, 44, 15-39.

Kupferman, L., (1991). L'aspect nominal et l'extraction en en. Le français moderne, 59, 113-147.

Lamiroy, B. (1999). Auxiliaires, langues romanes et grammaticalisation. Langages, 135, 33-45.

Larrivée, P. (2008). Qu'est-ce que la sémantique peut dire du sens lexical ? Langages, 172, 3-13.

Leeman, D. (2005). Relations anaphoriques et noms de parties du corps. La Syntaxe au cœur de la Grammaire. Presses universitaires de Rennes, 189-197.

Marque-Pucheu, C. (2008). La couleur des prépositions à et de. Langue française, 157, 74-105.

Mathieu, Y. Y. (1995). Verbes psychologiques et interprétation sémantique. Langue française, 105, 98-106.

Balibar-Mrabti, A. (1995). Une étude de la combinatoire des noms de sentiment dans une grammaire locale. Langue française, 105, 88-97.

Meunier, A. (1984). Une construction à prédicat nominal: Luc a l'audace de mentir à Léa. Lingvisticae Investigationes, Supplementa 9, 267-284.

Molinier, C. (1991). Les compléments adverbiaux du français de type avec N. Lingvisticae Investigationes, $X V, 115-140$.

Mostrov, V. (2015). L'être humain et la relation partie-tout, Les noms d'humains : une catégorie à part? Stuttgart : Franz Steiner Verlag, 115-146.

Muller, C. (2006). Portée négative et free choice dans les indéfinis de type que ce soit et n'importe. Langages, 162, 7-31.

Paykin, K., Tayalati, F. et Van de Velde, D. (2015). Les noms d'évaluation de comportement. Travaux de linguistique, 71 , 43-74.

Picoche, J. (1995). Combien y a-t-il de cœur(s) en français? Langue française, 105, 120-125.

Van de Velde, D. (1995). Le spectre nominal - des noms de matière aux noms d'abstraction. Leuven / Paris : Peeters.

Van Peteghem, M. (2006). Anaphores associatives intra-phrastiques et inaliénabilité. Aux carrefours du sens - Hommages offerts à Georges Kleiber. Leuven / Paris : Peeters, 441-456.

\footnotetext{
${ }^{1}$ Merci à nos relecteurs, leurs remarques nous ont permis de revoir le texte. Les erreurs restent bien sûr les nôtres.

${ }^{2}$ C'est le cas dans bien d'autres langues : on trouvera une « explication » de ce constat empirique dans Dingemanse (2009) et un panorama inter-linguistique documenté de cette variation sémantique dans Kraska-Szlenk (2014).

${ }^{3}$ Meunier (1984) et Paykin et al. (2015) étudient la même construction où un nom de qualité (cf. infra) prend la place du $\mathrm{N}_{\mathrm{pc}}$ (ce qui est probablement plus «classique ») : Luc a l'audace de mentir à Léa, Paul a la bonté d'aider Jean... Nous évoquons leurs travaux plus loin.

${ }^{4} \mathrm{C}$ 'est un raccourci : des bases de données, aussi vastes soient-elles, ne donnent qu'un échantillon d'attestations.

${ }^{5}$ Interrogeant ce qui peut porter sur la modalité, Gosselin (2015: 14-16) distingue les fonctionnements modaux de le tyran a eu la chance d'échapper aux flammes et le tyran a eu de la chance d'échapper aux flammes. Dans Meunier (1984: 275), on trouve déjà cette observation, également reprise dans Paykin et al. (2015). Nous exploiterons cette remarque valable pour avoir (de) la veine de dans une étude ultérieure.

${ }^{6}$ Cet emploi singulier peut sembler contestable. Cependant, nous ne l'écartons pas car le recours au singulier, là où le pluriel parait plus usité, pourrait trouver une explication (cf. 3.2.1, infra).
} 
${ }^{7}$ D'autres constructions du type avoir le $N$ à Vinf (avoir l'esprit à travailler / l'âme à rire) pourraient relever de cette polarité négative. Toutefois, comme elle caractérise également avoir le cœur de $V_{\text {inf }}$, nous réservons les remarques sur cette singularité à la section 3.3.1 consacrée à tête et cour.

${ }^{8}$ Nous admettons que cela est discutable (cf. cependant la note 9) du point de vue des modalités (aléthiques) logiques puisqu'il faudrait considérer les êtres humains ont un nez comme une phrase analytique (Anscombre, 2001 : 73). Mais, cela est vrai du point de vue des modalités (aléthiques) physiques génériques (cf. cependant la note 8). En effet, Gosselin rappelle qu'une proposition nécessaire du point de vue physique n'est pas forcément nécessaire du point de vue logique (Gosselin, 2015 : 25-29). Tout dépend du sous-système de valeurs modales aléthiques choisi. Ici, le choix du sous-système des modalités (aléthiques) physiques nous parait justifié par le fait que nous nous intéressons à l'acception (physiologique) du $\mathrm{N}_{\mathrm{pc}}$ et donc à ce que à quoi il réfère dans les contextes ad hoc.

${ }^{9}$ Ou presque nécessaire (il arrive qu'un être humain soit privé de nez) (cf. Gosselin et François, $1991: 69$ ).

${ }^{10}$ Selon Van de Velde, «il entre dans la définition même d'une table d'avoir des pieds » (1995: 159). Cette réflexion tend à faire de une table a des pieds une phrase analytique. En outre, à propos du syntagme la porte du réfrigérateur, Kupferman (1991: 120) note que de instaure une relation nécessaire entre porte et réfrigérateur (« dans notre univers de croyance usuel, le réfrigérateur a nécessairement une porte »).

${ }^{11}$ Cf. Flaux et Van de Velde (2000) ainsi que Van de Velde (1995) et Mostrov (2015). Nous distinguons les qualités des sentiments (et des émotions). Classiquement, les $\mathrm{N}$ de sentiment sont sélectionnés sur la base de leur compatibilité avec éprouver / ressentir ou un sentiment de (Balibar-Mrbati, 1995 ; Goossens, 2005) alors que les $\mathrm{N}$ de qualité entrent dans la construction faire preuve de (Molinier, 1991 : 130-132) : Max éprouve de la joie / *générosité vs Max fait preuve de *joie / générosité. Le N compassion, compatible avec les deux constructions : Max éprouve de la compassion / fare preuve de compassion à l'égard de Pierre, présente ainsi deux acceptions. Ces critères sont discutables et discutés (Anscombre, 1995). Dans Bertin (2018), aux qualités, nous opposons simplement les états (passions chez Aristote) qui comprennent (notamment) les sentiments et les émotions. Les états sont nécessaires sur le plan référentiel (tous les êtres humains sont joyeux/amoureux) mais contingents sur le plan temporel (un être humain est joyeux/amoureux à certains moments, pas à d'autres). Certains $\mathrm{N}_{\mathrm{pc}}$ donnent lieu à des acceptions ETAT, nous en donnons un exemple plus loin avec nerf (cf. 3.3.3).

${ }^{12}$ On notera avec Van de Velde (1995 : 167-169) qu'un emploi absolu n'est pas toujours possible avec un N de qualité. On peut opposer Paul a de l'audace / du courage à Paul a de la ?générosité/?gentillesse.

${ }^{13}$ Ces $\mathrm{N}_{\mathrm{pc}}$ sont toutefois difficilement compatibles avec faire preuve de (cf. note 11) : Paul fait preuve de cœur/*nez/*ventre vs Paul fait preuve de générosité/intuition/courage.

${ }^{14}$ Meunier qui étudie précisément cette construction (cf. note 3) d'un point de vue syntaxique cite l'exemple « métaphorique » suivant : Paul n'a pas eu les épaules de supporter cette épreuve (1984:278).

${ }^{15}$ Pour Meunier, si les constructions relèvent d'analyses différentes, le N garde une unité sémantique (1984: 276).

${ }^{16}$ Notre intuition de locuteur est ici confortée par l'absence de telles séquences dans Fx et fW. Cependant, nous avons trouvé cet énoncé attribué à l'Académie : C'est avoir bien du front ! (cité par le Grand Robert).

${ }^{17}$ C'est ce que le test avec faire preuve de a le mérite de mettre en avant (cf. note 11). Cette caractéristique rapproche les $\mathrm{N}$ de qualité des $\mathrm{N}$ d'attitude (Anscombre, 1995) et des $\mathrm{N}$ d'évaluation de comportement (NEC) étudiés par Paykin et al. (2015) en lien, précisément, avec des exemples comme Paul a la bonté de nous aider.

${ }^{18}$ L'énoncé Paul a de l'esprit est en revanche tout à fait acceptable. Mais, précisément, il s'agit alors d'un emploi QUAL pour un nom qui, " hors contexte », semble relever de la partie nécessaire - cf. Bertin (2018: 163-164) et Mostrov (2015: 135) qui oppose être d'un tempérament colérique à avoir du tempérament. Cf. également Mathieu (1995 : 101) qui regroupe esprit, âme, cour et æeil dans une unique catégorie notée $\mathrm{N}_{\mathrm{pc}}$.

${ }^{19}$ Un de nos relecteurs nous fait précisément remarquer - cf. Louis XIV avait /*a eu le nez bourbon - que cette opposition imparfait / passé composé recoupe l'opposition permanent / transitoire. Or cette opposition rejoint la distinction entre partie nécessaire (intrinsèque, permanente) et qualité contingente (transitoire).

${ }^{20} \mathrm{Cf}$. également Marque-Pucheu qui, comparant des constructions comme s'empresser à / de, observe que « d'une manière générale, l'engagement du sujet est plus fort dans les constructions en $\grave{a} »(2008: 102)$. Ainsi, avec $a ̀$, on se focalise sur le sujet qui n'est pas dans une certaine disposition alors que, avec de, on se focalise sur l'action elle-même. La portée de la négation le confirme : Paul n'avait pas le cœur à travailler mais il a travaillé quand même alors que Paul n'a pas eu le cœur de travailler implique que Paul n'a pas travaillé (cf. note 5).

${ }^{21}$ Comme nous le fait remarquer un relecteur, cette différence se retrouve avec verre à vin (propriété potentielle) vs. verre de vin (propriété effective).

${ }^{22}$ A la section 3.3.4, nous revenons sur avoir de l'œil dont le $P R$ donne un usage possible quoique très marginal.

${ }^{23}$ i.e. porteur d'une valeur modale appréciative positive. Les modalités appréciatives « consistent en une évaluation subjective du caractère désirable ou indésirable d'une situation ou d'un objet » (Gosselin, 2015 : 7).

${ }^{24}$ Et du nom familier couille (non sélectionné par nos critères de constitution du corpus initial). Le nom est compatible avec NPV : ah enfin un groupe de heavy qui a les couilles de chanter en français (fW). 